\title{
UNCONVENTIONAL FERMIONS: THE PRICE OF QUARK-LEPTON UNIFICATION AT TEV SCALES *
}

\author{
P. Q. HUNG \\ Dept. of Physics, University of Virginia, \\ 382 McCormick Road \\ P. O. Box 400714, Charlottesville, Virginia 22904-4714, USA \\ E-mail: pqh@virginia.edu
}

\begin{abstract}
The early petite unification (PUT) of quarks and leptons at $\mathrm{TeV}$ scales with $\sin ^{2} \theta_{W}\left(M_{Z}^{2}\right)$ used as a constraint, necessitates the introduction of extra quarks and leptons with unconventional electric charges (up to $4 / 3$ for the quarks and 2 for the leptons). This talk, in honor of Paul Frampton's 60th birthday, will be devoted to the motivation and construction of models of early unification and to their implications, including the issues of rare decays and unconventional fermions.
\end{abstract}

\section{Happy Birthday, Paul!}

\section{Motivations}

It is now believed that the Strong and Electroweak forces are very well described by $S U(3)_{c} \otimes S U(2)_{L} \otimes U(1)_{Y}$ at energies near or below the electroweak scale. It is also widely believed that the SM is just a low energy manifestation of some deeper unified theory which could explain why the three gauge couplings are so different, why the quantum numbers of the quarks are different from those of the leptons, and (wishfully thinking) why fermion masses are the way they are. Some of the most successful and popular unification scenarios are the quintessential $S U(5)$ or $S O(10)$ supplemented by supersymmetry. With 3 couplings $\alpha_{3}\left(M_{Z}^{2}\right)$, $\alpha_{2}\left(M_{Z}^{2}\right), \alpha^{\prime}\left(M_{Z}^{2}\right)$, one can make two predictions: $M_{G U T} \approx 10^{16} \mathrm{GeV}$ and $\sin ^{2} \theta_{W}\left(M_{Z}^{2}\right)=0.233(2)$.

Are there alternatives to GUT that can make predictions for $\sin ^{2} \theta_{W}\left(M_{Z}^{2}\right)$ and that can be tested? In particular, can these alterna-

* This work is supported in parts by the US Department of Energy under Grant No. DE-A505-89ER40518. 
tives be constructed for energy scales in the $\mathrm{TeV}$ region instead of being close to the Planck scale? These are well motivated questions which are enhanced by recent interests in the possibility that the "fundamental scale" lies in the $\mathrm{TeV}$ region, in the context of large extra dimensions. The key quantity used in the search for such alternatives is

$$
\sin ^{2} \theta_{W}\left(M_{Z}^{2}\right)=0.23113(15)
$$

which is very precisely measured.

Twenty two years ago, a construction of an alternative to GUT was made by Hung, Buras, and Bjorken ${ }^{1}$ based on the data available at the time, namely $\sin ^{2} \theta_{W}\left(M_{Z}^{2}\right) \sim 0.22$. The unification scale was found to be $\sim 1000 \mathrm{TeV}$, "small" enough to be coined the name Petite Unification (PUT) .

In light of the new and more precise data and of new theoretical motivations, a reexamination of PUT was performed by two of us (AB and $\mathrm{PQH})^{2}$ yielding three possible scenarios with some containing unconventional fermions. These three scenarios predict the PUT scale to be less than $10 \mathrm{TeV}$. What is most remarkable about two of the three scenarios is the existence of these unconventional fermions which provide a natural way for avoiding the severe constraint coming from the process $K_{L} \rightarrow \mu e$, as we shall see below.

Since the concept of large extra dimensions involves scales in the $\mathrm{TeV}$ range, it was natural to investigate the possibility of early unification within the LED context. This, in fact, has been done by Chacko, Hall, and Perelstein ${ }^{3}$ and by Dimopoulos and Kaplan ${ }^{4}$. The model used in ${ }^{3}$ was, in fact, one of the scenarios studied in ${ }^{1}$ transported to five dimensions.

\section{A petite review of Petite Unification}

\subsection{What is Petite Unification?}

In any unification scheme, one would like to know what the predictions might be. For example, in GUT, starting from three fundamental couplings: $g_{3}, g_{2}, g^{\prime}$, one obtains one fundamental coupling: $g_{G U T}$, which results in two predictions: $M_{G U T}$ and $\sin ^{2} \theta_{W}\left(M_{Z}^{2}\right)$.

For Petite Unification, starting from three fundamental couplings: $g_{3}, g_{2}, g^{\prime}$, one obtains two fundamental coupling: $g_{S}, g_{W}$, which results in one prediction: $\sin ^{2} \theta_{W}\left(M_{Z}^{2}\right)$ when the scale of Petite Unification is constrained independently. The requirement that the scale is less than 10 $\mathrm{TeV}$, for example, severely constrains the PUT gauge groups as we shall 
see below.

We assume the PUT gauge group to be $G=G_{S} \otimes G_{W}$ with the following pattern of symmetry breaking:

$$
G \stackrel{M}{\longrightarrow} G_{1} \stackrel{\tilde{M}}{\longrightarrow} G_{2} \stackrel{M_{Z}}{\longrightarrow} S U(3)_{c} \otimes U(1)_{E M},
$$

where

$$
G_{1}=S U(3)_{c}\left(g_{3}\right) \otimes \tilde{G}_{S}\left(\tilde{g}_{S}\right) \otimes G_{W}\left(g_{W}\right),
$$

and

$$
G_{2}=S U(3)_{c}\left(g_{3}\right) \otimes S U(2)_{L}\left(g_{2}\right) \otimes U(1)_{Y}\left(g^{\prime}\right) .
$$

It turns out that the most economical choices for $G_{S}$ and $G_{W}$ are the Pati-Salam $S U(4)_{P S}{ }^{5}$ and $S U(N)^{k}$ respectively. The PUT group is now

$$
G=S U(4)_{P S} \otimes S U(N)^{k},
$$

with a permutation symmetry assumed so that each $S U(N)$ of $S U(N)^{k}$ has the same gauge coupling.

The next task is to compute $\sin ^{2} \theta_{W}\left(M_{Z}^{2}\right)$. Using $Q=T_{3 L}+T_{0}$ and $T_{0}=$ $\sum_{\alpha} C_{\alpha W} T_{\alpha W}^{0}+C_{S} T_{15}$, and the matching of the electromagnetic coupling with the weak couplings at $M_{Z}$, one arrives at the following master formula for $\sin ^{2} \theta_{W}\left(M_{Z}^{2}\right)^{1}$ :

$$
\begin{aligned}
\sin ^{2} \theta_{W}\left(M_{Z}^{2}\right)= & \sin ^{2} \theta_{W}^{0}\left[1-C_{S}^{2} \frac{\alpha\left(M_{Z}^{2}\right)}{\alpha_{S}\left(M_{Z}^{2}\right)}-8 \pi \times\right. \\
& \left.\alpha\left(M_{Z}^{2}\right)\left(K \ln \frac{\tilde{M}}{M_{Z}}+K^{\prime} \ln \frac{M}{\tilde{M}}\right)\right],
\end{aligned}
$$

where $\alpha\left(M_{Z}^{2}\right) \equiv e^{2}\left(M_{Z}^{2}\right) / 4 \pi, \quad \alpha_{S}\left(M_{Z}^{2}\right) \equiv g_{3}^{2}\left(M_{Z}^{2}\right) / 4 \pi$, and

$$
\sin ^{2} \theta_{W}^{0}=\frac{1}{1+C_{W}^{2}}
$$

with $C_{W}^{2}=\sum_{\alpha} C_{\alpha W}^{2}$. Here

$$
\begin{gathered}
K=b_{1}-C_{W}^{2} b_{2}-C_{S}^{2} b_{3}, \\
K^{\prime}=C_{S}^{2}\left(\tilde{b}-\tilde{b}_{3}\right) .
\end{gathered}
$$

$\tilde{b}$ and $\tilde{b}_{3}$ are the one-loop Renomalization Group coefficients, above $\tilde{M}$, of $U(1)_{S}$ and $S U(3)_{c}$ respectively. Furthermore, the following (fairly) precise inputs are used in Eq. 6: $1 / \alpha\left(M_{Z}^{2}\right)=127.934(27), \alpha_{S}\left(M_{Z}^{2}\right)=0.1172(20)$.

From Eq. 6, it is important to realize the following point: If we require $M$ and $\tilde{M}$ to be at most $10 \mathrm{TeV}$, the logarithmic evolution of $\sin ^{2} \theta_{W}$ is 
less important than in the GUT case. In our case, a term which is crucial in the determination of $\sin ^{2} \theta_{W}$ is the following term in Eq. 6: $C_{S}^{2} \frac{\alpha\left(M_{Z}^{2}\right)}{\alpha_{S}\left(M_{Z}^{2}\right)}$. This can easily be seen by looking at $\sin ^{2} \theta_{W}\left(M_{Z}^{2}\right)=\sin ^{2} \theta_{W}^{0}\left(1-0.067 C_{S}^{2}-\right.$ $\log$ terms $)$. Indeed, $\sin ^{2} \theta_{W}\left(M_{Z}^{2}\right)$ is found to be very sensitive to the value of $C_{S}^{2}$ which, as we shall see, depends crucially on the fermion representation under $G_{W}$. Since we have already chose $G_{S}$ to be $S U(4)_{P S}$, the choices of $G_{W}$ amount to their predictions for $\sin ^{2} \theta_{W}^{0}$ and the choices of fermion representations as represented by $C_{S}^{2}$.

Our objectives are therefore the computations of $\sin ^{2} \theta_{W}^{0}$ and $C_{S}^{2}$, and to examine the related physical consequences.

\section{2. $\sin ^{2} \theta_{W}^{0}$ and $C_{S}^{2}$}

The computation of $\sin ^{2} \theta_{W}^{0}$ was done in detail in ${ }^{1}$ and repeated in ${ }^{2}$. Here, I will simply state the results.

By the definition of $\sin ^{2} \theta_{W}^{0}$ as shown in Eq. 7, its computation requires simply the adjoint representation of $G_{W} \cdot{ }^{1}$ arrived at the following important constraint: Only weak gauge bosons (i.e. the gauge bosons of $G_{W}$ ) with charges $0, \pm 1$ are consistent with the data. We obtained a very simple formula for $\sin ^{2} \theta_{W}^{0}$ :

$$
\sin ^{2} \theta_{W}^{0}=\frac{N}{\left.k T r\left(Q_{W}^{2}\right)\right|_{a d j}}=\frac{N}{k n_{1}}=\frac{N}{2 k r_{0}\left(N-r_{0}\right)},
$$

with $n_{1}$ is the number of weak gauge bosons with charges \pm 1 and $n_{1}=$ $2 r_{0} r_{1}$, with $[\underbrace{\tilde{Q}_{W}, \cdots \tilde{Q}_{W}}_{r_{0}}, \underbrace{\tilde{Q}_{W}-1, \cdots \tilde{Q}_{W}-1}_{r_{1}}]$. The results for $\sin ^{2} \theta_{W}^{0}$ are listed in Table 1 below.

Since $\tilde{Q}_{W}^{i}=\frac{1}{4}\left(3 Q_{q}^{i}+Q_{l}^{i}\right)$, one can see that only groups and representations with $\tilde{Q}_{W}^{i}= \pm \frac{1}{2}$ or $\tilde{Q}_{W}^{i}=0, \pm 1$ can accomodate standard fermions. With this in mind, Table 2 gives the values of $C_{S}^{2}$ along with the corresponding quark and lepton charges.

From Table 1 and 2 , and from $\sin ^{2} \theta_{W}\left(M_{Z}^{2}\right)=\sin ^{2} \theta_{W}^{0}\left(1-0.067 C_{S}^{2}-\right.$ $\log$ terms), it is clear that groups with "high" $\sin ^{2} \theta_{W}^{0}$ need "high" $C_{S}^{2}$.

A close examination revealed three favorite candidates for $G_{W}$ :

1. $[S U(2)]^{4}: C_{S}^{2}=2 / 3 ; \sin ^{2} \theta_{W}^{0}=0.25$.

This group contains only conventionally-charged quarks and leptons since the fermion representations under $G_{W}$ are of the type $(f, 1, .$.$) as$ one can easily infer from Tables 1 and 2 .

2. $[S U(2)]^{3}, \quad[S U(3)]^{2}: C_{S}^{2}=8 / 3 ; \sin ^{2} \theta_{W}^{0}=1 / 3,3 / 8$. 
Table 1. The values of $\sin ^{2} \theta^{0}$ for the weak groups $G_{W}=S U(N)^{k}$ and different fermion representations.

\begin{tabular}{ccccc}
\hline$G_{W}$ & $r_{0}$ & $\sin ^{2} \theta_{W}^{0}$ & $(f, 1)+(1, f)$ & $(f, f)$ \\
\hline$[S U(2)]^{3}$ & 1 & 0.333 & $\pm \frac{1}{2}$ & $0, \pm 1$ \\
{$[S U(2)]^{4}$} & 1 & 0.250 & $\pm \frac{1}{2}$ & $0, \pm 1$ \\
{$[S U(3)]^{2}$} & 1 & 0.375 & $\frac{2}{3},-\frac{1}{3}$ & $0, \pm 1$ \\
{$[S U(3)]^{3}$} & 1 & 0.250 & $\frac{2}{3},-\frac{1}{3}$ & $0, \pm 1$ \\
{$[S U(4)]^{2}$} & 2 & 0.250 & $\pm \frac{1}{2}$ & $0, \pm 1$ \\
{$[S U(5)]^{2}$} & 1 & 0.313 & $\frac{4}{5},-\frac{1}{5}$ & $0, \pm 1$ \\
{$[S U(6)]^{2}$} & 1 & 0.300 & $\frac{5}{6},-\frac{1}{6}$ & $0, \pm 1$ \\
$S U(7)$ & 3 & 0.292 & $\frac{4}{7},-\frac{3}{7}$ & \\
{$[S U(7)]^{2}$} & 1 & 0.292 & $\frac{6}{7},-\frac{1}{7}$ & $0, \pm 1$ \\
$S U(8)$ & 3 & 0.267 & $\frac{5}{8},-\frac{3}{8}$ & \\
$S U(8)$ & 4 & 0.250 & $\pm \frac{1}{2}$ & \\
\hline
\end{tabular}

Table 2. The values of lepton $\left(Q_{l}^{i}\right)$ and quark $\left(Q_{q}^{i}\right)$ electric charges and the corresponding weak charge $\left(\tilde{Q}_{W}^{i}\right)$, and $C_{S}^{2}$.

\begin{tabular}{cccc}
\hline$Q_{W}^{i}$ & $Q_{l}^{i}$ & $Q_{q}^{i}$ & $C_{S}^{2}$ \\
\hline$\frac{1}{2}$ & 0 & $\frac{2}{3}$ & \\
$-\frac{1}{2}$ & -1 & $-\frac{1}{3}$ & \\
$\frac{1}{2}$ & 1 & $\frac{1}{3}$ & $\frac{2}{3}$ \\
$-\frac{1}{2}$ & 0 & $-\frac{2}{3}$ & \\
\hline 1 & 0 & $\frac{4}{3}$ & \\
0 & -1 & $\frac{1}{3}$ & \\
-1 & -2 & $-\frac{2}{3}$ & $\frac{8}{3}$ \\
1 & 2 & $\frac{2}{3}$ & \\
0 & 1 & $-\frac{1}{3}$ & \\
-1 & 0 & $-\frac{4}{3}$ & \\
\hline
\end{tabular}

These groups contain conventionally-charged quarks and leptons as well as unconventional quarks and leptons with higher charges $\pm 4 / 3$ and \pm 2 as can be seen from Table 2 . The $G_{W}$-fermion representations are of the types: $(f, \bar{f}, .$.$) .$

The use of the term "favorite" actually means that these are the three groups that can give $\sin ^{2} \theta_{W}\left(M_{Z}^{2}\right)$ within the allowed experimental range for unification scales which are less than $10 \mathrm{TeV}$. We shall see however that $[S U(2)]^{4}$ suffers from problems with rare decays, and we will be left with $[S U(2)]^{3}, \quad[S U(3)]^{2}$ as the true favorites. 
In order to calculate $\sin ^{2} \theta_{W}\left(M_{Z}^{2}\right)$ or, equivalently, the unification scale $M$, a knowledge of at least the fermionic degrees of freedom that enter the evolution of $\sin ^{2} \theta_{W}$ is necessary.

\section{Unconventional Fermions}

Although this section is titled "unconventional fermions", I will list the fermion contents of all three "favorite" candidates.

I) $P U T_{0}=S U(4)_{\mathrm{PS}} \otimes S U(2)_{L} \otimes S U(2)_{R} \otimes S \tilde{U}(2)_{L} \otimes S \tilde{U}(2)_{R}:$

- Standard Fermions: $\Psi_{L}=\left(q_{L}, l_{L}\right)=(4,2,1,1,1)_{L}, \Psi_{R}=$ $\left(q_{R}, l_{R}\right)=(4,1,2,1,1)_{R}$.

- Fermions of "Mirror Group": $\tilde{\Psi}_{L}=\left(\tilde{q}_{L}, \tilde{l}_{L}\right)=(4,1,1,2,1)_{L}, \tilde{\Psi}_{R}=$ $\left(\tilde{q}_{R}, \tilde{l}_{R}\right)=(4,1,1,1,2)_{R}$.

It should be understood that the adjective "Mirror" refers to something completely different (groups instead of fermions) from its customary use in Left-Right symmetric models.

II) $P U T_{1}=S U(4)_{\mathrm{PS}} \otimes S U(2)_{L} \otimes S U(2)_{H} \otimes S U(2)_{R}$ :

Below the electric charges of the fermions are explicitely written down in parentheses next to their names.

- Standard Fermions:

$$
\psi_{L, R}^{q}=\left(\begin{array}{c}
u(2 / 3) \\
d(-1 / 3)
\end{array}\right)_{L, R} ; \psi_{L, R}^{l}=\left(\begin{array}{c}
\nu(0) \\
l(-1)
\end{array}\right)_{L, R}
$$

- Fermions with "weird" charges:

$$
\tilde{Q}_{L, R}=\left(\begin{array}{l}
\tilde{U}(4 / 3) \\
\tilde{D}(1 / 3)
\end{array}\right)_{L, R} ; \tilde{L}_{L, R}=\left(\begin{array}{l}
\tilde{l}_{u}(-1) \\
\tilde{l}_{d}(-2)
\end{array}\right)_{L, R}
$$

- Fermion Representations:

$(4,2,2,1)_{L}$

$\left[\left(i \tau_{2} \psi_{L}^{q, *}, \tilde{Q}_{L}\right),\left(\tilde{L}_{L}, \psi_{L}^{l}\right)\right] ;(4,1,2,2)_{R}=\left[\left(i \tau_{2} \psi_{R}^{q, *}, \tilde{Q}_{R}\right),\left(\tilde{L}_{R}, \psi_{R}^{l}\right)\right] ;$

$(4,2,1,1)_{L, R}=\left[\tilde{Q}_{L, R}^{\prime}, \tilde{L}_{L, R}^{\prime}\right] ;(4,1,1,2)_{L, R}=\left[\tilde{Q}_{L, R}^{\prime \prime}, \tilde{L}_{L, R}^{\prime \prime}\right]$

- Tree-level $S U(2)_{H}$ transition:

$$
\begin{aligned}
& i \tau_{2} \psi_{L}^{q, *} \rightarrow \tilde{Q}_{L} \\
& \tilde{L}_{L} \rightarrow \psi_{L}^{l}
\end{aligned}
$$

$\Rightarrow$ No tree-level transition between normal quarks and leptons due to $S U(2)_{H}$ gauge bosons

- Tree-level $S U(4) / S U(3)_{c} \otimes U(1)_{S}$ transition:

$$
\begin{aligned}
& i \tau_{2} \psi_{L}^{q, *} \rightarrow \tilde{L}_{L} \\
& \tilde{Q}_{L} \rightarrow \psi_{L}^{l} \\
& \text { etc... }
\end{aligned}
$$


$\Rightarrow$ No tree-level transition between normal quarks and leptons due to PS gauge bosons.

III) $P U T_{2}=S U(4)_{\mathrm{PS}} \otimes S U(3)_{L} \otimes S U(3)_{H}:$

- Fermion Representations of the type $(4,3, \overline{3})$ and $(4, \overline{3}, 3)$.

- Same presence of higher charged fermions as in $P U T_{1}$ !

Is the presence of quarks and leptons with unconventional charges in $P U T_{1,2}$ a boon or a bane? As we shall see below, the existence of these fermions turns into a virtue for $P U T_{1,2}$ when we look at the decay process $K_{L} \rightarrow \mu^{ \pm} e^{\mp}$. Before discussing the virtues and defects of these three scenarios, let us do some RG analysis to see the range of values that the unification scales can take.

\section{RG analysis and PUT scales}

- If $M$ and $\tilde{M}$ are of $\mathrm{O}(\mathrm{TeV})$, there is not much "running" to do starting from $M_{Z}$. This means that two-loop contributions to $\sin ^{2} \theta_{W}\left(M_{Z}^{2}\right)$ are not as important as the one-loop contribution. A detailed RG analysis up to two loops within the context of $P U T_{1}$ will be presented in ${ }^{6}$. Here I will neglect that contribution for simplicity as we had done in ${ }^{2}$.

- To find PUT scales from $\sin ^{2} \theta_{W}\left(M_{Z}^{2}\right)$ in our RG analysis, we assume the unconventional fermions to have a mass $M_{F}=(250 \pm$ 50) $\mathrm{GeV}$. Furthermore, we assume all vector-like fermions (present in $\left.P U T_{1,2}\right)$ to have a mass of order $M$.

- Let us start out with $\tilde{M}=M$. We then use Eq. (1), namely $\sin ^{2} \theta_{W}\left(M_{Z}^{2}\right)=0.23113(15)$, as a constraint to obtain $M$. Since the scale of $S U(2)_{R} \otimes S \tilde{U}(2)_{L} \otimes S \tilde{U}(2)_{R}$ breaking is naturally of order $M$, we require (from the constraint on $W_{R}$ ) that $M \geq 800 \mathrm{GeV}$. Furthermore, we also require $M_{F} \geq 200 \mathrm{GeV}$. We obtained the following results shown in Figure 1.

- One can also look at the case where $\tilde{M} \neq M$. This is shown in Figure 2 below.

The following conclusions arise by examining Figure 1. 1) For $P U T_{0}$, one needs the number of generations $n_{G} \geq 9$ for the SM and $n_{G} \geq 4$ for MSSM. 2) For $P U T_{1}$ with $n_{G}=3$, one obtains $M=(1.00 \pm 0.14)$. 3) For $\mathrm{PUT}_{2}$ with $n_{G}=3$, one obtains $M=(3.30 \pm 0.47) \mathrm{TeV}$. 


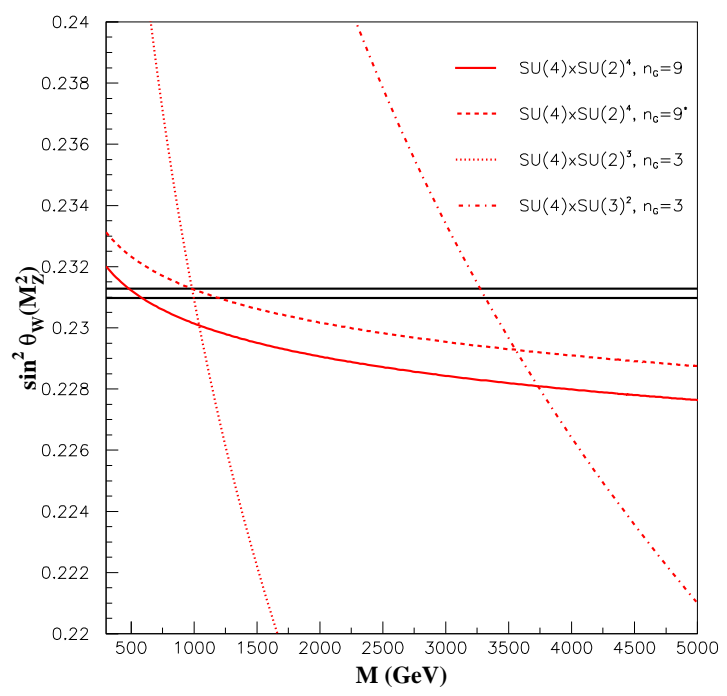

Figure 1. $\sin ^{2} \theta_{W}\left(M_{Z}^{2}\right)$ versus the PUT scale $M$. The horizonal band represents the experimental value. The dashed curve $\left(n_{G}=9^{*}\right)$ is obtained by using $M_{F}=200 \mathrm{GeV}$, while the other three curves are obtained by using $M_{F}=250 \mathrm{GeV}$.

From the above analysis, one can see that the PUT scales are all below $10 \mathrm{TeV}$ as promised.

\section{Virtues and Defects}

I will now discuss in particular the defects of $P U T_{0}$ and the virtues of the unconventional fermions in $P U T_{1,2}$.

- $P U T_{0}$ :

The defects are the following:

1) Large number of generations! (On the other hand, why not?)

2) Tree-level transition between SM fermions which leads to a large $\operatorname{Br}\left(K_{L} \rightarrow \mu e\right)$.

$\operatorname{Br}\left(K_{L} \rightarrow \mu^{ \pm} e^{\mp}\right)=4.7 \cdot 10^{-12}\left(\frac{\alpha_{S}\left(m_{G}\right)}{0.1}\right)^{2} \times\left[\frac{1.8 \cdot 10^{3} \mathrm{TeV}}{m_{G}}\right]^{4}$

versus

$\operatorname{Br}\left(K_{L} \rightarrow \mu e\right)<4.7 \times 10^{-12}$

Since $m_{G} \sim M<1 \mathrm{TeV}$, the bound is violated by at least 13 orders of magnitude!

Chacko, Hall and Perelstein ${ }^{3}$ solved this problem by taking $P U T_{0}$ into 


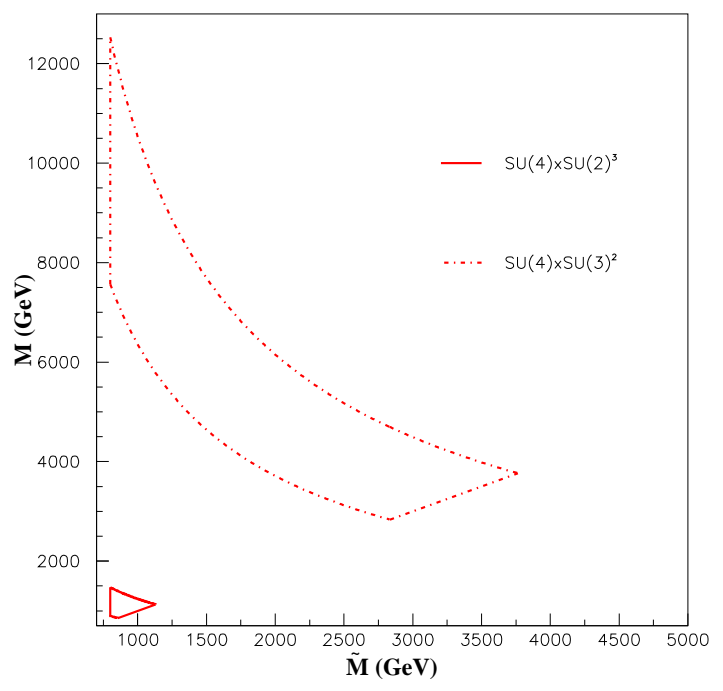

Figure 2. The allowed ranges (at $2 \sigma$ ) for the $S U(2)^{3}$ and $S U(3)^{2}$ scenarios.

five dimensions.

- $P U T_{1}\left(P U T_{2}\right)$ :

The virtues of the unconventional fermions are as follows.

1) Tree-level transitions via $S U(2)_{H}$ and $S U(4) / S U(3)_{c} \otimes U(1)_{S}$ gauge bosons only occur between unconventional and normal fermions. There is no tree-level FCNC.

The process $K_{L} \rightarrow \mu e$ occurs in box diagrams and can be made small! (Exactly zero when the unconventional fermions in the boxes are made degenerate.)

2) The lightest of the unconventional fermions (quark or lepton) is unstable. It can decay entirely into normal fermions since the Higgs sector for the model can mix $W_{L}, W_{R}, W_{H}$. For example, if $\tilde{l}_{u}(-1)$ were the lightest of such particles (still presumably having a mass larger than $M_{W}$ ), it can have the decay mode $\tilde{l}_{u}(-1) \rightarrow \nu W$. The rate will depend on the details of the mixing of the gauge bosons ${ }^{6}$.

3) Since the lightest one is unstable, there is no cosmological constraint.

4) Fermions such as these unconventional ones can be searched for at the LHC (see ${ }^{7}$ ), especially if they are relatively 'long lived". 


\section{Conclusions}

From our analysis, we have arrived at two favorite models: $P U T_{1}$ and $P U T_{2}$. (A detailed study of $P U T_{1}$ will appear in the very near future ${ }^{6}$.) The correct $\sin ^{2} \theta_{W}\left(M_{Z}^{2}\right)$ was obtained for a PUT scale from 1-10 TeV. These models predict an absence of tree-level FCNC because of the presence of unconventionally charged quarks and leptons: The tree-level transitions only connect these fermions to the normal ones! Heavy (less than $1 \mathrm{TeV}$ ) and perhaps "long lived" quarkonic or leptonic unconventional fermions are characteristic signatures of this model. Is this a heavy price to pay for early unification or an actual bonus? Last but not least, since the unification scale is in the low $\mathrm{TeV}$ region, one might wonder if there is any link to the physics of large extra dimensions.

\section{Acknowledgments}

I wish to thank Paul Frampton and the organizers for a wonderful conference. Our thoughts go to Behram Kursunoglu who tirelessly organized the Coral Gables series until he ultimately left us in October 2003.

\section{References}

1. P. Q. Hung, A. J. Buras and J. D. Bjorken, Phys. Rev. D 25, 805 (1982).

2. A. J. Buras and P. Q. Hung, Phys. Rev. D 68, 035015 (2003).

3. Z. Chacko, L. J. Hall, and M. Perelstein, JHEP 0301, 001 (2003).

4. S. Dimopoulos and D. E. Kaplan, Phys. Lett. B 531, 127 (2002).

5. J. C. Pati and A. Salam, Phys. Rev. D 10, 275 (1974).

6. A. J. Buras, P. Q. Hung, N. K. Tran, A. Poschenrieder, and E. Wyszomirski, "Early $S U(4)_{\mathrm{PS}} \otimes S U(2)_{L} \otimes S U(2)_{R} \otimes S U(2)_{H}$ Unification of Quarks and Leptons", hep-ph/0406048.

7. P. H. Frampton, P. Q. Hung, and M. Sher, Phys. Rep. 330, 263 (2000). 